\title{
Specificity of the Redox Complex between Cytochrome P450 24A1 and Adrenodoxin Relies on Carbon-25 Hydroxylation of Vitamin-D Substrate ${ }^{\text {田 }}$
}

\author{
Amit Kumar and D. Fernando Estrada \\ Department of Biochemistry, Jacobs School of Medicine and Biomedical Science, University at Buffalo, Buffalo New York
}

Received April 26, 2019; accepted July 1, 2019

\section{ABSTRACT}

Metabolic deactivation of $1,25(\mathrm{OH})_{2} \mathrm{D} 3$ is initiated by modification of the vitamin-D side chain, as carried out by the mitochondrial cytochrome P450 24A1 (CYP24A1). In addition to its role in vitaminD metabolism, CYP24A1 is involved in catabolism of vitamin-D analogs, thereby reducing their efficacy. CYP24A1 function relies on electron transfer from the soluble ferredoxin protein adrenodoxin (Adx). Recent structural evidence suggests that regioselectivity of the CYP24A1 reaction may correlate with distinct modes of Adx recognition. Here we used nuclear magnetic resonance (NMR) spectroscopy to monitor the structure of ${ }^{15} \mathrm{~N}$-labeled full-length Adx from rat while forming the complex with rat CYP24A1 in the ligand-free state or bound to either $1,25(\mathrm{OH})_{2} \mathrm{D} 3$ or the vitamin-D supplement $1 \alpha(\mathrm{OH}) \mathrm{D} 3$. Although both vitamin-D ligands were found to induce a reduction in overall NMR peak broadening, thereby suggesting ligand-induced disruption of the complex, a crosslinking analysis suggested that ligand does not have a significant effect on the relative association affinities of the redox complexes. However, a key finding is that, whereas the presence of primary CYP24A1 substrate was found to induce NMR peak broadening focused on the putative recognition site $\alpha$-helix 3 of rat adrenodoxin, the interaction in the presence of $1 \alpha(\mathrm{OH}) \mathrm{D} 3$, which is lacking the carbon-25 hydroxyl, results in disruption of the NMR peak broadening pattern, thus indicating a ligand-induced nonspecific protein interaction. These findings provide a structural basis for the poor substrate turnover of side-chain-modified vitamin-D analogs, while also confirming that specificity of the CYP24A1-ligand interaction influences specificity of CYP24A1-Adx recognition.

\section{SIGNIFICANCE STATEMENT}

Mitochondrial cytochrome P450 enzymes, such as CYP24A1 responsible for catabolizing vitamin-D and its analogs, rely on a protein-protein interaction with a ferredoxin in order to receive delivery of the electrons required for catalysis. In this study, we demonstrate that this protein interaction is influenced by the enzyme-ligand interaction that precedes it. Specifically, vitaminD missing carbon-25 hydroxylation binds the enzyme active site with high affinity but results in a loss of P450-ferredoxin binding specificity.

\section{Introduction}

The bioactive form of vitamin-D, $1,25(\mathrm{OH})_{2} \mathrm{D} 3$, is responsible for the maintenance of calcium and phosphate homeostasis, with insufficiency of the hormone causing vitamin-D-dependent rickets and osteomalacia, among other diseases (Holick, 2007; Ryan et al., 2013). The metabolic stability of $1,25(\mathrm{OH})_{2} \mathrm{D} 3$ relies on the activity of the multifunctional mitochondrial enzyme CYP24A1, which initiates deactivation of the hormone by mediating modification of the $1,25(\mathrm{OH})_{2} \mathrm{D} 3$ side chain to produce either carbon-23 (C23) or carbon-24 (C24) hydroxylation products (Siu-Caldera et al., 1995; Beckman et al., 1996). Although the human isoform is both a C23 and C24 hydroxylase, in other species

This work was supported by the National Institutes of Health Institute of General Medical Sciences [Grant no. R00GM112862 (D.F.E.)]. The authors have no conflict of interest to declare.

https://doi.org/10.1124/dmd.119.087759.

S This article has supplemental material available at dmd.aspetjournals.org. regioselectivity is divided between either the C23 or C24 pathway (Hamamoto et al., 2006; Prosser et al., 2007). Subsequent activity by CYP24A1 includes catalysis of all ensuing steps for each pathway to produce either calcitroic acid $(\mathrm{C} 24)$ or the vitamin-D receptor antagonist $1,25(\mathrm{OH})_{2} \mathrm{D} 3-26,23$-lactone $(\mathrm{C} 23)$. Owing to its central role in vitaminD metabolism, the function of CYP24A1 is a primary concern in the development of vitamin-D analogs designed to therapeutically treat vitamin-D deficiency. The ideal analog must maintain signaling activity via its interaction with the vitamin-D receptor yet must also be catalytically resistant (therefore metabolically stable) with regard to modification by CYP24A1.

Like most cytochrome P450 enzymes (P450s), CYP24A1 catalysis requires sequential transfer of two external electrons to reduce the heme iron and produce the reactive Fe(IV)oxo intermediate. In mitochondria, these reduction steps necessitate an interaction with the water-soluble iron-sulfur [2Fe-2S] protein adrenodoxin (Adx) (Ewen et al., 2011). We recently reported an analysis by nuclear magnetic resonance (NMR) spectroscopy of the interaction between CYP24A1 and Adx, in which

ABBREVIATIONS: Adx, rat adrenodoxin; CYP24A1, cytochrome P450 24A1; EDC, 1-ethyl-3-(3-dimethylaminopropyl)carbodiimide; [2Fe-2S], iron sulfur cluster; HSQC, heteronuclear single quantum coherence; NMR, nuclear magnetic resonance; P450, cytochrome P450. 


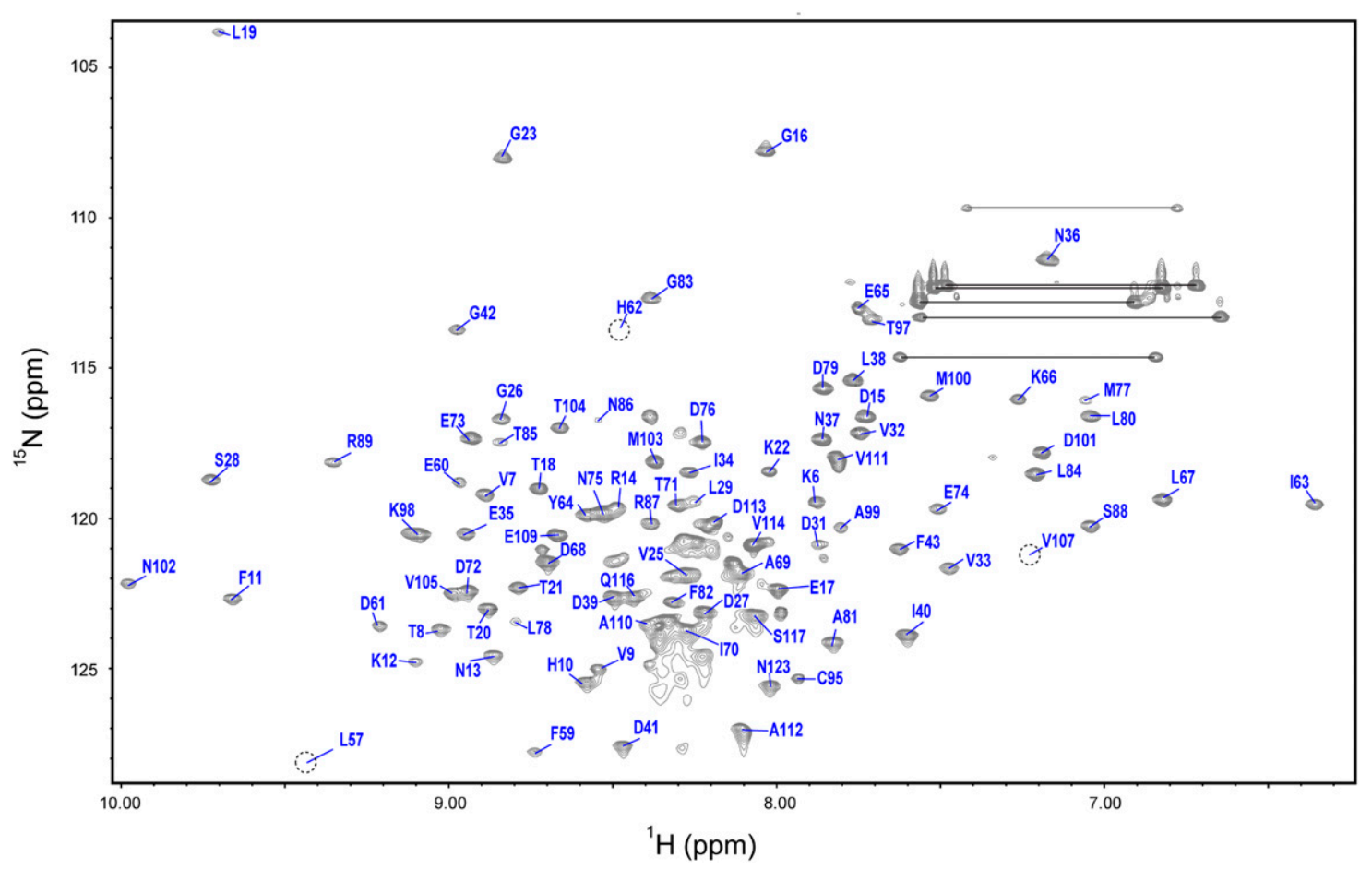

Fig. 1. Assigned ${ }^{15} \mathrm{~N}-\mathrm{HSQC}$ spectrum of oxidized full-length rat Adx.

clotrimazole-bound CYP24A1 isoforms from species with different regioselectivity for $1,25(\mathrm{OH})_{2} \mathrm{D} 3$ were found to rely on distinct secondary contacts for the protein-protein complex (Estrada, 2018). This finding suggested a correlation between site-specific regioselective hydroxylation of the substrate with recognition of the redox partner; we hypothesized that this feature would require structural communication between the active site and the opposite (proximal) surface of the enzyme.

In the present study, we sought to confirm the existence of substrateinduced two-way communication between the active site and the Adx recognition surface of CYP24A1. We monitored the protein interaction using two-dimensional NMR and by incorporating ligand-free CYP24A1 or CYP24A1 bound to either $1,25(\mathrm{OH})_{2} \mathrm{D} 3$ or the vitamin-D supplement $1 \alpha(\mathrm{OH}) \mathrm{D} 3.1 \alpha(\mathrm{OH}) \mathrm{D} 3$ is of particular interest as a functional probe because it retains high affinity for CYP24A1 yet is an extremely poor substrate compared with $1,25(\mathrm{OH})_{2} \mathrm{D} 3$ (Kaufmann et al., 2011). Comparative mapping of the peak-broadening pattern from the ${ }^{15} \mathrm{~N}$-Adx signal demonstrated that $1,25(\mathrm{OH})_{2} \mathrm{D} 3$ enhances the specificity of the interaction, as reflected by focused mapping of the interaction on $\alpha$-helix 3 of rat adrenodoxin, but that the absence of a carbon- 25 hydroxyl from $1 \alpha(\mathrm{OH}) \mathrm{D} 3$ makes the interaction structurally and functionally nonspecific. These findings represent direct biophysical evidence of substrate-driven allostery in a critical vitamin-D-metabolizing P450.

\section{Materials and Methods}

Protein Expression and Purification. The gene encoding full-length rat Adx (GenBank accession number NP_05882.1) was modified to contain a C-terminal polyhistidine tag and was custom-synthesized (Genscript) and cloned into a pET$15 \mathrm{~b}$ expression vector (Novagen). Overexpression and purification of ${ }^{15} \mathrm{~N}-\mathrm{Adx}$ was carried out as described previously (Estrada, 2018). Backbone assignment of Adx required ${ }^{15} \mathrm{~N}$ and ${ }^{13} \mathrm{C}$ labeling, which was carried out by supplementing minimal growth media with $4 \mathrm{~g} / \mathrm{l}$ of ${ }^{13} \mathrm{C}$ glucose and $1 \mathrm{~g} / \mathrm{l}$ of ${ }^{15} \mathrm{~N}$ ammonium chloride (Cambridge Isotope). Purity was determined by a $\mathrm{A}_{415} / \mathrm{A}_{280}$ ratio greater than 0.8. Sample concentration was calculated using an extinction coefficient for the iron cofactor of $11 \mathrm{mM}^{-1} \mathrm{~cm}^{-1}$. Single-residue mutations of rat Adx were also generated by Genscript and were purified identically to wild-type protein.
Generation of recombinant rat CYP24A1 (GenBank accession number NP_963966.1) was carried out as in our previous protocol (Estrada, 2018) with the following modifications. Once CYP24A1 was bound to a saturated Adx-affinity Ni NTA column, the column was washed using five column volumes of equilibration buffer (10 $\mathrm{mM}$ potassium phosphate, 20\% glycerol, $0.1 \%$ 3-[(3-cholamidopropyl) dimethylammonio]-1-propanesulfonate (CHAPS), $2 \mathrm{mM} \quad \beta$-mercaptoethanol, $\mathrm{pH}$ 7.4) followed by five column volumes at $30 \%$ elution buffer $(500 \mathrm{mM}$ potassium phosphate, $300 \mathrm{mM} \mathrm{NaCl}, 20 \%$ glycerol, $0.1 \%$ CHAPS, $2 \mathrm{mM}$ $\beta$-mercaptoethanol, $\mathrm{pH}$ 7.4). The protein was then recovered using $100 \%$ elution buffer, in which the high salt concentration effectively disrupted the CYP24A1Adx interaction of the Adx-affinity column. Subsequent purification by gel filtration was carried out in the same affinity elution buffer and produced a protein purity ratio $\left(\mathrm{A}_{417} / \mathrm{A}_{280}\right)$ greater than 1.0 (Supplemental Fig. 7A).

NMR Assignment and Homology Modeling of Adx. Data acquisitions of two- and three-dimensional experiments were carried out at $25^{\circ} \mathrm{C}$ on a Varian Inova $600-\mathrm{MHz}$ spectrometer equipped with a cryogenic probe. A sample of ${ }^{15} \mathrm{~N}$ and ${ }^{13} \mathrm{C}$ labeled Adx was exchanged into NMR buffer consisting of $50 \mathrm{mM}$ potassium phosphate ( $\mathrm{pH}$ 6.8) and $50 \mathrm{mM} \mathrm{NaCl}, 10 \% \mathrm{D}_{2} \mathrm{O}$, and was brought to a $2.6 \mathrm{mM}$ protein concentration. Data sets used to assign the protein backbone consisted of heteronuclear single quantum coherence (HSQC), HNCA, HNCACB, and HNCOCA experiments (Bax and Ikura, 1991; Grzesiek et al., 1992, 1993; Constantine et al., 1993). The data were processed using nmrPipe (Delaglio et al., 1995) and the processed spectra were analyzed on NMRViewJ (Johnson, 2004). The ${ }^{15} \mathrm{~N}$ and ${ }^{13} \mathrm{C}$ chemical shift values determined during assignment of the protein have been submitted to the Biologic Magnetic Resonance Data Bank (BMRB) (ID number 27673). Secondary chemical shift values $(\mathrm{C} \alpha)$ were used to predict secondary structural elements (Wishart and Sykes, 1994). Owing to the high degree of similarity between chemical shift predictions of the backbone secondary structure with that of the reported crystal structure of bovine Adx (Pikuleva et al., 2000), the bovine structure (PDB ID ICJE) was used as a template for homology modeling of rat Adx using the I-TASSER and SWISS-MODEL servers (Yang et al., 2015; Waterhouse et al., 2018). The modeled Adx structure was then validated using PROCHECK (Laskowski et al., 1996).

Spectral Ligand Binding Assays. Binding of calcitriol or alfacalcidol (both from ApexBio) to CYP24A1 was carried out as described previously with minor modifications (Estrada, 2018). Briefly, ligand binding was measured by monitoring perturbation of the Soret peak at incremental concentrations of ligand. 


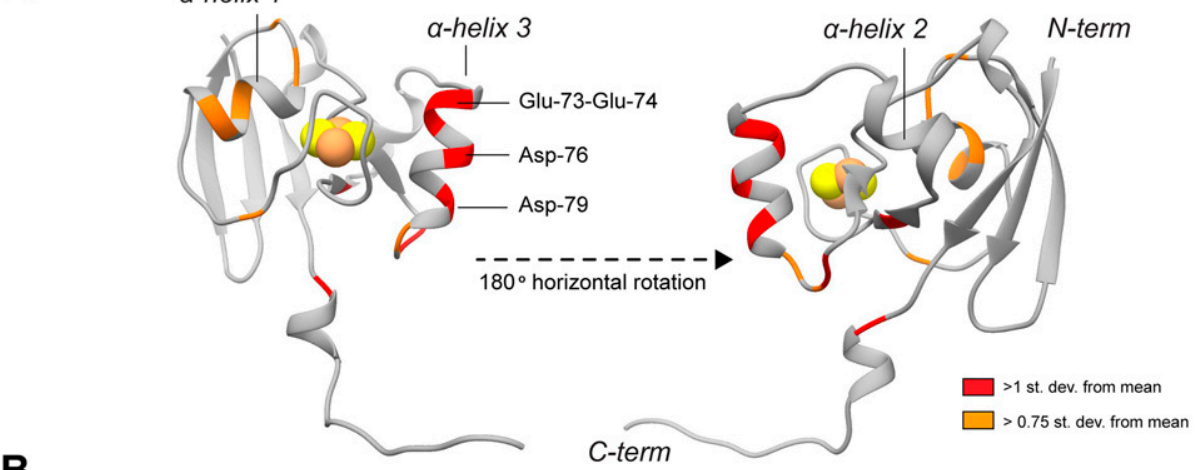

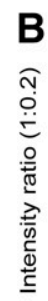

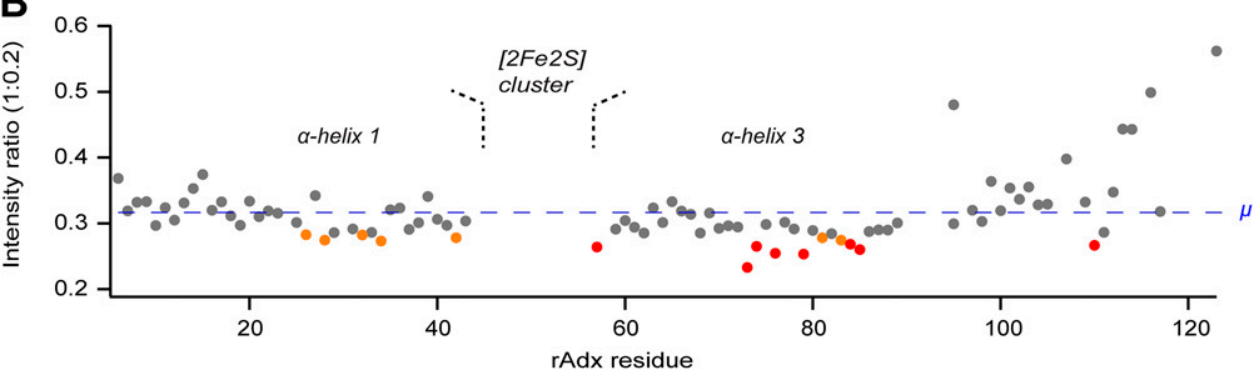

Fig. 2. Mapping of differential peak broadening of ${ }^{15} \mathrm{~N}$-Adx. (A) The structure of Adx was modeled on the basis of the secondary chemical shift index. Regions that undergo differential peak broadening as a result of titration with $0.2 \mathrm{M}$ equivalents of ligand-free CYP24A1 are indicated by red ( 1 S.D.) and orange $(0.75$ S.D.). (B) Corresponding peak broadening for all assigned residues, with the mean ratio indicated by the dashed line.
Binding assays were carried out in triplicate with CYP24A1 in gel filtration buffer, concentrated to $10 \mathrm{mg} / \mathrm{ml}$, then diluted to $1 \mu \mathrm{M}$ in $100 \mathrm{mM}$ potassium phosphate, $\mathrm{pH}$ 7.4. Ligand titrations (between 0 and $8 \mu \mathrm{M}$ ) were added from a dimethyl sulfoxide (DMSO) stock with total DMSO contributing to $1.6 \%$ of total sample volume. At each concentration, the enzyme and ligand were incubated for 12 minutes at $25^{\circ} \mathrm{C}$ prior to recording of absorption spectra on a Shimadzu UV-2700 spectrophotometer. Baseline-subtracted difference spectra showing a maximum and a minimum at 385 and $418 \mathrm{~nm}$ were used to generate total spectral response, which was then plotted against ligand concentration to generate total binding data. Binding constants were then calculated upon fitting data using Prism 7.02 (GraphPad software) to the saturation binding equation with ligand depletion shown below, in which $a=-1.0, b=K d+X+B_{\max }$, and $c=-1 \bullet X \bullet B_{\max }$ and $X=$ total ligand added.

$$
\text { Total binding }=-b+\sqrt{ }\left(b^{2}-4 \bullet a \bullet c\right) /(2 \bullet a)
$$

NMR Titration of ${ }^{15} \mathrm{~N}$-Adx with CYP24A1. All ${ }^{15} \mathrm{~N}$-HSQC spectra were acquired using a final concentration of $100 \mu \mathrm{M}$ of ${ }^{15} \mathrm{~N}$-Adx. As described earlier (Estrada, 2018), a specific sequence of steps for buffer exchange was carefully followed to ensure that extraneous unbound detergent was removed from CYP24A1 samples prior to combining with $50 \mathrm{nmol}$ of ${ }^{15} \mathrm{~N}$-Adx. Briefly, CYP24A1 was first exchanged into a high-salt, detergent-free buffer by a 10 -fold volume exchange through a $50-\mathrm{kDa}$ filter (Amicon), then combined with ${ }^{15} \mathrm{~N}-\mathrm{Adx}(100 \mu \mathrm{M})$ and exchanged into a low-salt NMR sample buffer by a 10-fold volume exchange through a $10 \mathrm{kDa}$ filter. NMR spectra of mutant ${ }^{15} \mathrm{~N}$-Adx were acquired using the same protocol. As observed previously, the stoichiometric excess of ${ }^{15}$ N-Adx to CYP24A1 (1:0.2 or 1:0.4) conferred stability on the P450. Following each data acquisition, each sample was recovered and the P450 quantified spectrophotometrically, with no significant loss of CYP24A1 detected. Samples containing either $1,25(\mathrm{OH})_{2} \mathrm{D} 3$ or $1 \alpha(\mathrm{OH}) \mathrm{D} 3$ were prepared using the same procedure but with an excess concentration of ligand (50 $\mu \mathrm{M}$ ligand combined with 20 or $40 \mu \mathrm{M}$ CYP24A1) introduced during exchange into NMR buffer. The absorption spectra of ligand-bound CYP24A1 samples were monitored upon completion of data collection (Supplemental Fig. 1) to ensure that ligand remained bound during NMR data acquisition.

Each spectrum was acquired using 96 scans and 128 increments, with a total acquisition time of 4.5 hours per experiment. The peak intensities corresponding to each assigned amide were quantified from the processed spectra and the values expressed as a ratio of remaining intensity using the following formula:

$$
I_{\text {remaining }}=I_{C Y P \text { added }} / I_{\text {free }}
$$

From these values, differential peak broadening was identified using either 0.75 or 1 full S.D. from the mean of the remaining intensities. For representations of net peak broadening across the entire Adx structure, individual residue peak ratios were treated as separate data points and graphed in box plots. Box plots were generated using Interactive Dotplot (http://statistika.mfub.bg.ac.rs/interactivedotplot/) (Weissgerber et al., 2017).

Chemical Crosslinking. Purified rat CYP24A1 was incubated with rat Adx under low-salt conditions (10 mM potassium phosphate buffer, $\mathrm{pH} 7.4)$ and in the presence of the zero-length crosslinker 1-ethyl-3-(3-dimethylaminopropyl)carbodiimide (EDC) (Thermo Scientific). To compare crosslinking data with complex formation by NMR, the protein ratios were kept consistent with those used for NMR binding data. Specifically, each reaction was run in triplicate using 8 and $16 \mu \mathrm{M}$ ligand-bound and unliganded P450 corresponding to 1:0.2 and 1:0.4 ratios, respectively, combined with $40 \mu \mathrm{M} \mathrm{Adx}$ in a $20-\mu 1$ reaction volume. After a 2-hour incubation period at $25^{\circ} \mathrm{C}$, the reaction was terminated by addition of an equal volume of Laemmli loading dye. The samples were then resolved by gel electrophoresis and stained with Coomassie Blue. The protein bands were quantified by Image Laboratory 6.0 (Bio-Rad). Crosslinking efficiency was calculated as a ratio of band volume of the crosslinked product divided by total band volume of the complex, CYP24A1, and Adx combined. Saturation curves were obtained by titrating CYP24A1 between 0 and $20 \mu \mathrm{M}$ against a fixed concentration of $40 \mu \mathrm{M} \mathrm{Adx}$ and quantified as a ratio of the volume of the complex band to that of a loading control of CYP24A1 alone. The curves were fitted using a one-site specific binding equation in Prism 5.0 (GraphPad).

CYP24A1 Functional Assays. Reconstituted CYP24A1 assays were carried out in 100- $\mu$ l volumes and contained either equal concentrations (each $1.5 \mu \mathrm{M}$ ) of rat CYP24A1, rat Adx, and bovine adrenodoxin reductase, or reduced CYP24A1 to match the protein ratios captured by NMR. $1,25(\mathrm{OH})_{2} \mathrm{D} 3$ and $1 \alpha(\mathrm{OH}) \mathrm{D} 3$ were kept constant at $5 \mu \mathrm{M}$. The reaction mixtures were first preincubated for 5 minutes at $37^{\circ} \mathrm{C}$, then initiated by addition of NADPH to a final concentration of $1 \mathrm{mM}$. After a further 5 -minute incubation at $37^{\circ} \mathrm{C}$, the reactions were terminated by addition of two volumes of methanol. Internal standards were then added to a final concentration of $2 \mu \mathrm{M} .25(\mathrm{OH}) \mathrm{D} 3$ was used as an internal standard for reactions containing $1,25(\mathrm{OH})_{2} \mathrm{D} 3$ as substrate, and $1,25(\mathrm{OH})_{2} \mathrm{D} 3$ was used as an internal standard for reactions containing $1 \alpha(\mathrm{OH}) \mathrm{D} 3$ as substrate. Samples were subjected 
to centrifugation at $34,000 \mathrm{~g}$ for 30 minutes and $150 \mu \mathrm{l}$ of the supernatant was removed and diluted with additional methanol to a final volume of $200 \mu \mathrm{l}$. Highperformance liquid chromatography samples were resolved on an Agilent 1260 Infinity II liquid chromatography system with a Poroshell 120 EC-C18 column $(4.6 \times 250 \mathrm{~mm})$ and using an isocratic mobile phase of $70 \%$ acetonitrile, $30 \%$ water. Elution peaks were detected by monitoring absorbance at $264 \mathrm{~nm}$. All samples were run in quadruplicate and the remaining substrate for each was calculated as a percentage of substrate measured from a control sample in which CYP24A1 was withheld. Accumulation of $\mathrm{H}_{2} \mathrm{O}_{2}$ was measured in quadruplicate using a colorimetric assay (Quantitative Peroxide Assay Kit, no. 23280; Pierce), with a serial dilution of a $30 \% \mathrm{H}_{2} \mathrm{O}_{2}$ solution used as a standard curve.

A

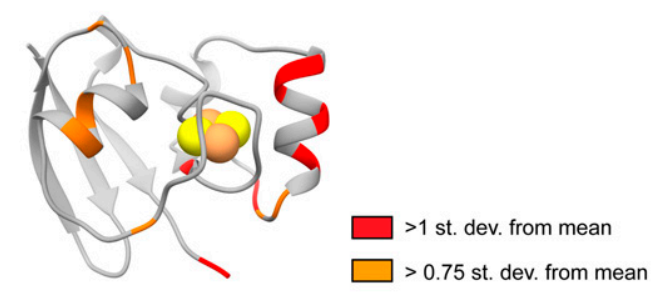

rat Adx wt
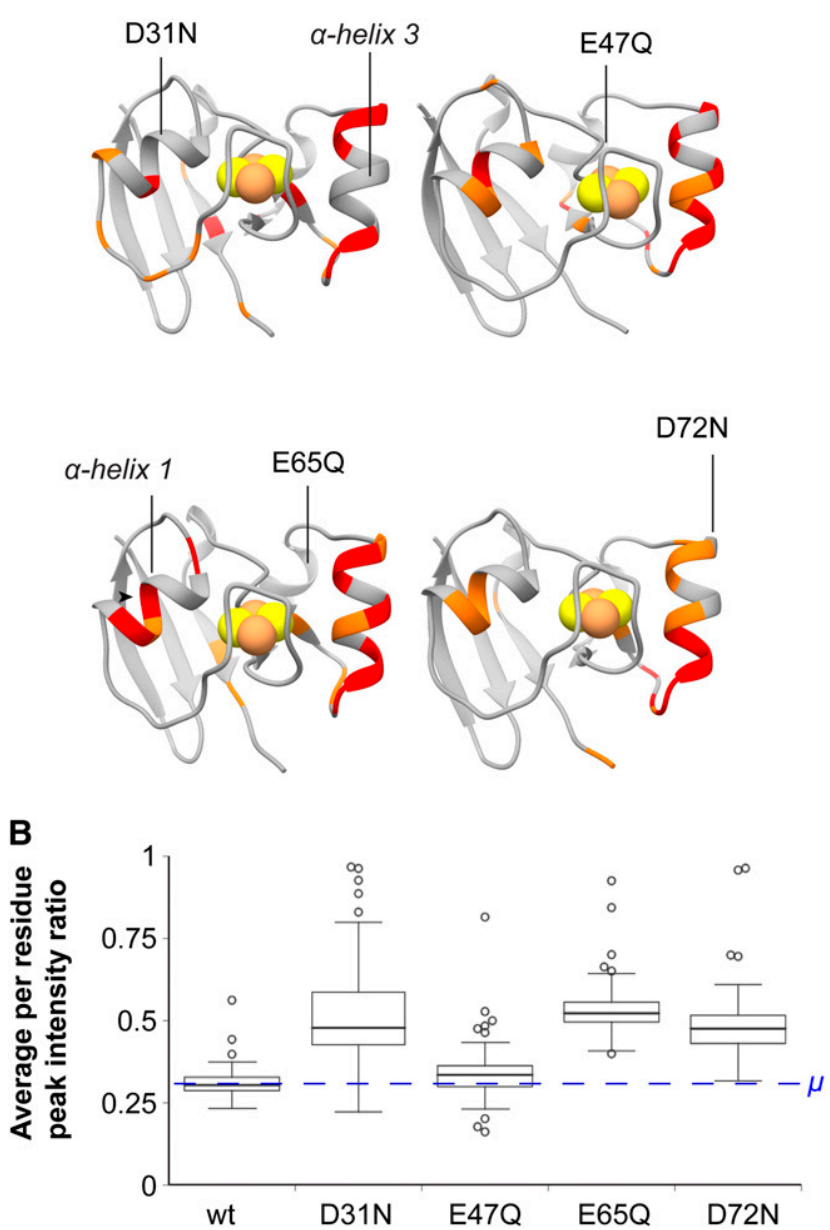

Fig. 3. Effect of charge-neutralizing mutations on peak broadening of ${ }^{15} \mathrm{~N}$-Adx. (A) Point substitutions designed to neutralize surface charges on Adx perturb the CYP24A1-induced peak-broadening pattern to varying extents compared with wildtype protein. Differentially broadened residues are selected with 1 as a basis and 0.75 S.D. from the mean for a 1:0.2 ratio of ${ }^{15} \mathrm{~N}$-Adx/ligand-free CYP24A1. (B) Net per residue peak broadening for the interaction with ligand-free CYP24A1 is reflected in box plots in which remaining intensities for individual residues are represented by each data point, suggesting that mutations D31N, E65Q, and D72N all reduce the extent of the redox complex formation.

\section{Results}

NMR Assignment and Homology Modeling of Rat Adx. Recombinant human and bovine Adx have previously been characterized by NMR (Weiss et al., 2000; Kostic et al., 2002). However, to pair redox partners from the same species, we generated and characterized full-length (residues 1-128) ${ }^{15} \mathrm{~N}$-labeled Adx from rat, which shares approximately $85 \%$ sequence identity with bovine or human Adx. The ${ }^{15} \mathrm{~N}$-HSQC spectrum of Adx is well dispersed with resolvable amide peaks and presents a two-dimensional pattern similar to that of other species (Fig. 1). However, owing to ambiguities in transferring backbone chemical shift assignments from the BMRB database entries for related proteins, particularly in the crowded regions of the spectrum, the Adx NMR spectrum was assigned ab initio by analysis of three-dimensional correlation experiments (HNCA, HNCACB, and HNCOCA). Overall, approximately $70 \%$ of the backbone amides of Adx were assigned, including residues for all three short helices of the core domain, with notable exceptions for residues located near the paramagnetic $[2 \mathrm{Fe}-2 \mathrm{~S}]$ coordination loop.

Secondary chemical-shift values derived from NMR assignment of the $\alpha$ and $\beta$ carbons of residue side chains (defined as $\Delta \delta=$ $\delta_{\text {observed }}-\delta_{\text {random coil }}$ ) were used to predict the secondary structural elements of Adx (Wishart and Sykes, 1994). As expected, the secondary structure of rat Adx was found to align closely with that of bovine Adx (Supplemental Fig. 2), suggesting a similar overall protein fold. Therefore, bovine Adx [PDB 1CJE, (Pikuleva et al., 2000)] was used as a template for structure prediction of rat Adx via I-TASSER (Yang et al., 2015). The modeled structure (Fig. 2A) conforms to the conserved Adx fold, with a core domain consisting of three short $\alpha$-helices framing a solvent-exposed [2Fe-2S] cluster.

Interaction of ${ }^{15} \mathrm{~N}$-Adx with Ligand Free CYP24A1. To establish the baseline redox partner interaction in the absence of ligand, we acquired two-dimensional HSQC spectra of ${ }^{15} \mathrm{~N}$-Adx in the presence of increasing concentrations of ligand-free CYP24A1. Like our previous binding data acquired with the clotrimazole-bound CYP24A1, unliganded CYP24A1 also induced considerable broadening of the NMR signal, with approximately $32 \%$ of the original signal intensity remaining upon addition of 0.2 equivalents of CYP24A1 (Fig. 2B; Supplemental Fig. 3). However, unlike the interaction with the inhibitorbound enzyme, unliganded CYP24A1 produced a modest amount of differential line broadening in which specific amide signals are differentially broadened. This broadening of signal was probably the result of a combination of factors, including a protein interaction that undergoes intermediate chemical exchange, observed for protein complexes with dissociation constants between 1 and $10 \mu \mathrm{M}$, as well as an increase in the relative size of the observable ${ }^{15} \mathrm{~N}$-Adx when incorporated into the complex. However, differential broadening of NMR signal as a reporter of localized changes in the chemical environment has previously been demonstrated to correlate with functionally relevant P450 protein-protein binding interfaces (Estrada et al., 2013; Bart and Scott, 2017). Next, we mapped sites that were broadened more than 1 S.D. from the mean in response to addition of unliganded CYP24A1 (Fig. 2, highlighted in red). The pattern presents as a contiguous surface on the solvent-exposed side of $\alpha$-helix 3 composed of Glu-73, Glu-74, Asp-76, and Asp-79. The interaction with ligand-free CYP24A1 was also observed to produce a secondary broadening effect on $\alpha$-helix 1 (Fig. 2A, highlighted in orange).

Effects of Charge-Neutralizing Mutations of ${ }^{15} \mathrm{~N}-\mathrm{Adx}$ on Recognition of Ligand-Free CYP24A1. We designed a series of charge-neutralizing mutations in $\mathrm{Adx}$ as a way to assess contributions from particular anionic surface residues toward the redox interaction. Particular sites were selected for mutation on the basis of their proximity 
A

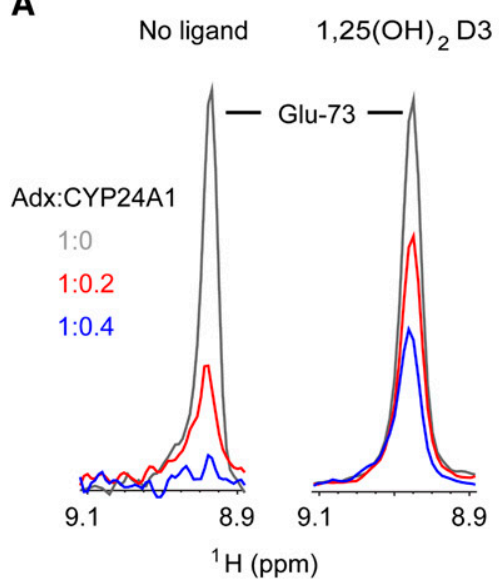

B

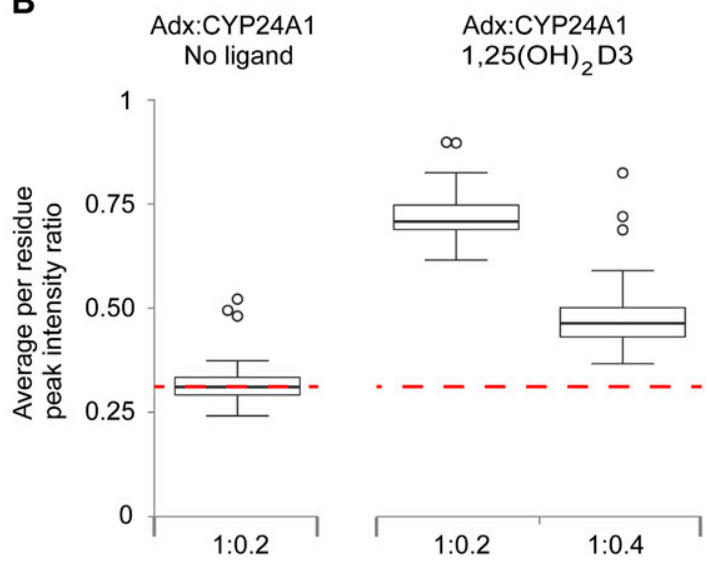

Adx:CYP24A1 $1 \mathrm{a}(\mathrm{OH}) \mathrm{D} 3$

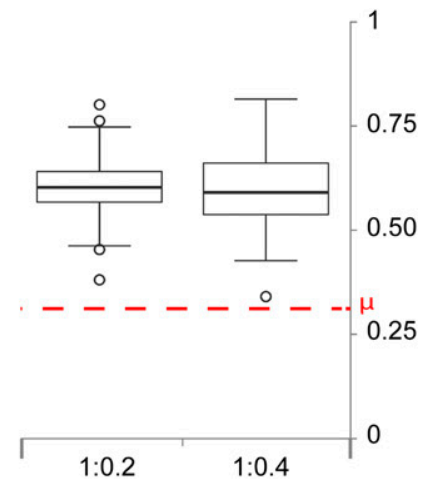

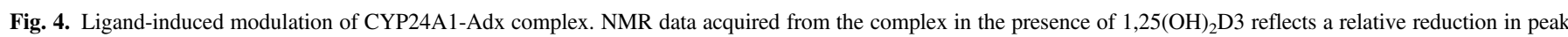

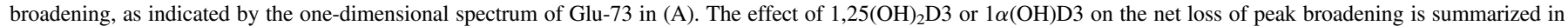
(B), in which remaining intensities for individual residues are represented by each data point in the box plots.

to the iron center, and therefore their anticipated involvement in recognition of CYP24A1. Further consideration was given to the distribution of the mutations, with substitutions located on each of the adjacent helices. As such, substitutions were carried out at each of $\alpha$-helices 1, 2, and 3 (D31N, E65Q, and D72N, respectively) as well as E47Q on the [2Fe-2S] coordinating loop. The structural integrity of each mutant was verified by overlaying the ${ }^{15} \mathrm{~N}$-HSQC spectra with that of wild-type ${ }^{15} \mathrm{~N}$-Adx (Supplemental Fig. 4). Although some mutations expectedly induced minor or modest chemical shift perturbations in the spectrum of ${ }^{15} \mathrm{~N}$-Adx, none of the mutations disrupted the overall protein fold.

We next compared NMR spectra of free ${ }^{15} \mathrm{~N}$-Adx mutants with spectra of the mutants in the presence of 0.2 equivalents of CYP24A1 (Fig. 3A; Supplemental Table 1). We observed that substituting individual anionic side chains with uncharged polar side chains does not abolish the complex. Rather, we observe subtle redistribution of peak broadening for the ${ }^{15} \mathrm{~N}$-Adx mutants. For example, the redox complex with mutation $\mathrm{D} 72 \mathrm{~N}$ on $\alpha$-helix 3 results in a local redistribution of peak broadening toward the $\mathrm{C}$-terminal end of the helix, probably owing to minor changes in complementary charge pairing to compensate for the charge removal ( $\alpha$-helix 3 contains multiple conserved anionic surfaces). In contrast, the mutant $\mathrm{E} 47 \mathrm{Q}$, designed to remove the sole charge on the $[2 \mathrm{Fe}-2 \mathrm{~S}]$ coordinating loop, displays a peak-broadening pattern very similar to that of wild-type.

Interestingly, mutations on $\alpha$-helices 1 and 2 result in more pronounced changes in the broadening pattern. The mutant E65Q on $\alpha$-helix 2 induces enhanced peak broadening at Val-32 of $\alpha$-helix 1 . In contrast, the mutant D31N on $\alpha$-helix 1 induces a reduction of peak broadening on Asp-76 of $\alpha$-helix 3. A further analysis of net line broadening for each mutant ${ }^{15} \mathrm{~N}$-Adx spectra with CYP24A1 indicated that certain mutations of the Adx surface reduce overall complex formation (Fig. 3B). These include the mutant at the expected recognition site, D72N, but also secondary sites like D31N and E65Q.

Mutational Effects on the ${ }^{15} \mathrm{~N}$ HSQC Spectrum of ${ }^{15} \mathrm{~N}$-Adx. Although none of the surface mutations of ${ }^{15} \mathrm{~N}-\mathrm{Adx}$ were found to compromise the protein fold, certain substitutions were found to perturb the ${ }^{15} \mathrm{~N}$-HSQC spectra in different ways. For example, D72N, in the absence of any P450, was found to induce only local changes in the amide signal for neighboring residues. Such "neighboring" effects, also observed for the E65Q mutation, are expected for substitution of a single side chain. In contrast, the mutant D31N, also in the absence of any $\mathrm{P} 450$, was found to induce both local as well as longer range perturbations on more remote sites, specifically on residues Val-7 to His10 on the first $\mathrm{N}$-terminal $\beta$-strand and on residue Lys- 98 of an adjacent loop (Supplemental Fig. 5).

Recognition of ${ }^{15} \mathrm{~N}$-Adx by CYP24A1 in Complex with $1,25(\mathrm{OH})_{2} \mathrm{D3}$. To examine the influence of $1,25(\mathrm{OH})_{2} \mathrm{D} 3$ on formation of the Adx-CYP24A1 complex, we acquired HSQC spectra of ${ }^{15} \mathrm{~N}$-Adx samples containing incremental concentrations of substratebound CYP24A1. To ensure that substrate would not directly affect the structure of Adx, we first overlaid spectra of ${ }^{15} \mathrm{~N}$-Adx with and without substrate and in the absence of P450. For this control experiment, we identified a small number of up-field chemical shift perturbations corresponding to the $\mathrm{N}$-terminal $\beta$-strands and the $\mathrm{C}$-terminal domain of Adx. However, the effects were generally distributed away from the three $\alpha$-helices near the iron and did not affect the peak intensity of the spectra. A subsequent comparison of ${ }^{15} \mathrm{~N}$-Adx with and without $1 \alpha(\mathrm{OH})$ D3 produced what we interpret to be similar nonspecific effects.

Interestingly, compared with the ligand-free titration, addition of 0.2 equivalents of substrate-bound CYP24A 1 did not produce the same degree of overall peak broadening. Final peak intensities were approximately twice as high as those observed in the ligand-free state for the corresponding protein ratios. Upon further addition (0.4 equivalents), the substrate-bound enzyme induced further broadening of the Adx spectrum but still not to the same degree as observed with 0.2 equivalents of ligand-free enzyme (Fig. 4A; Supplemental Fig. 6; Supplemental Table 2). Despite this reduction in the net peak broadening, addition of 0.4 equivalents of substratebound CYP24A1 induced a distribution of peak broadening on Adx that is more focused near $\alpha$-helix 3 compared with the pattern observed in the absence of substrate (Fig. 5, left and center panels). More focused peak broadening near $\alpha$-helix 3 also affects Leu-80, which has previously been reported as contributing a hydrophobic contact upon binding to CYP11B1 and CYP11B2 (Peng and Auchus, 2017).

$1 \alpha(O H) D 3$ Disrupts Specificity of the CYP24A1-Adx Redox Complex. To determine whether specificity of ligand binding influences recognition of $\mathrm{Adx}$, we acquired a parallel set of ${ }^{15} \mathrm{~N}$-Adx spectra in the presence of incremental concentrations of CYP24A1 bound to the analog $1 \alpha(\mathrm{OH}) \mathrm{D} 3$. Despite the absence of the carbon-25 hydroxyl (Fig. 6), titration of CYP24A1 with the analog produces a standard typeI shift of the Soret band toward $392 \mathrm{~nm}$. In general, spectral binding of $1 \alpha(\mathrm{OH}) \mathrm{D} 3$ appears comparable to that of $1,25(\mathrm{OH})_{2} \mathrm{D} 3$, with each displaying sub-micromolar affinity. Here we observe that addition of CYP24A1 bound to $1 \alpha(\mathrm{OH}) \mathrm{D} 3$ results in reduced net peak 


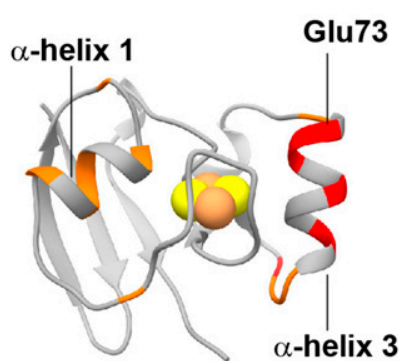
Adx:CYP24A1 (1:0.2)
No ligand

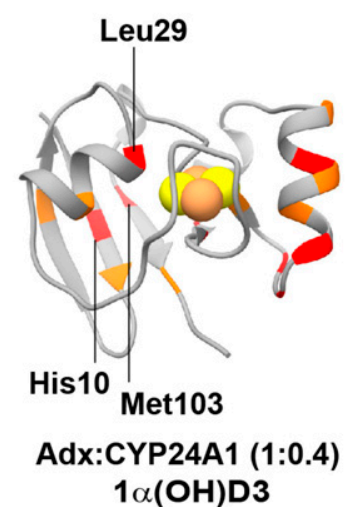

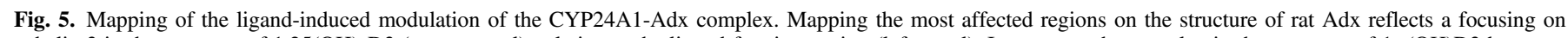

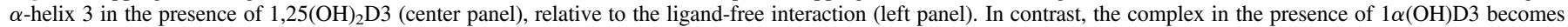
nonspecific, as indicated by increased distribution of peak broadening (right panel).

broadening (Fig. 4B). However, samples containing additional CYP24A1 ( 0.4 equivalents) fail to promote further complex formation, probably indicating a fundamental change in molecular recognition of Adx. Moreover, a comparison of the resulting peak broadening on $\mathrm{Adx}$ in response to the presence of both vitamin-D ligands demonstrates a significant disruption of the pattern when CYP24A1 is bound to $1 \alpha(\mathrm{OH}) \mathrm{D} 3$ (Fig. 5, right panel; Supplemental Fig. 6). Notably, the differential effect on $\alpha$-helix 3 is reduced, replaced by peak broadening distributed noncontiguously throughout the structure and affecting, among other sites, Leu-29 of $\alpha$-helix 1, His-10 and Met-103 of the anterior $\beta$-strands, and His-62 of $\alpha$-helix 2 .

Crosslinking of the CYP24A1-Adx Complex in the Presence of $1,25(\mathrm{OH})_{2} \mathrm{D3}$ and $1 \alpha(\mathrm{OH}) \mathrm{D3}$. Independently of the pattern of NMR peak broadening for individual residues, the presence of either ligand reduced overall net broadening, consistent with a disruption of the redox complex (Fig. 4). To evaluate this effect using a complementary approach, we carried out a series of chemical crosslinking experiments using the amine-reactive linker EDC. EDC has previously been used to capture redox complexes between the mitochondrial CYP11B enzymes and Adx (Peng and Auchus, 2017). EDC treatment of CYP24A1 with Adx followed by electrophoresis produces a resolvable protein complex band at approximately $65 \mathrm{kDa}$ (Fig. 7A; Supplemental Fig. 7). To draw a close comparison with the NMR data, the EDC reactions were carried out using protein ratios identical to those represented in the NMR data (1:0.2 and 1:0.4 for Adx: CYP24A1) and in the absence and presence of either ligand. Here we observe that there appear to be no significant differences between the crosslinking efficiency of the redox complex using CYP24A1 with or without ligand or bound to either $1,25(\mathrm{OH})_{2} \mathrm{D} 3$ or $1 \alpha(\mathrm{OH}) \mathrm{D} 3$ (Fig. 7B). As a way to quantify a relative affinity between each redox complex, we also carried out EDC crosslinking at incrementally increasing concentrations of CYP24A1. The saturation curves, shown in separate panels in Fig. 7C, indicate that the complex using unliganded CYP24A1 reaches a maximum at approximately $10 \mu \mathrm{M}$ CYP24A1 for samples containing $40 \mu \mathrm{M}$ Adx. We should note that accumulation of the covalently crosslinked product probably affects dissociation dynamics in a way that prevents measurement of full binding equilibria. However, the saturation curve suggests an apparent association constant $\left(K_{\text {on }}\right)$ of approximately $1.5 \mu \mathrm{M}$. This would be consistent with a binding constant for a protein interaction that displays the intermediate chemical exchange that we observe by NMR. Notably, addition of $1,25(\mathrm{OH})_{2} \mathrm{D} 3$ and $1 \alpha(\mathrm{OH}) \mathrm{D} 3$ produce similar saturation points, thus indicating that, as captured by chemical crosslinking, vitamin-D ligand does not induce a large change in binding affinity.

CYP24A1 Activity in the Presence of $1,25(\mathrm{OH})_{2} \mathrm{D3}$ and $1 \alpha(\mathrm{OH})$ D3 Results in Similar Production of $\mathbf{H}_{2} \mathrm{O}_{2}$. Production of $\mathrm{H}_{2} \mathrm{O}_{2}$ by way of a peroxide shunt is a well documented byproduct of $\mathrm{P} 450$ catalysis. In some cases, close coupling between reducing equivalents and productive catalysis is accompanied by a reduction in relative production of $\mathrm{H}_{2} \mathrm{O}_{2}$ (Peng et al., 2016), as more equivalents are routed to product formation. Here we anticipated that the low CYP24A1 activity resulting primarily from an unproductive orientation of substrate, and in which molecular recognition of the electron donor remains unaffected, would result in a proportional increase in the amount of reactive oxygen species produced as a result of unaltered electron delivery. As expected, in comparison with the biologic substrate, there was minimal depletion of $1 \alpha(\mathrm{OH}) \mathrm{D} 3$ either at a 1:1 Adx-to-CYP24A1 ratio (Fig. 8A) or at the ratios represented in the NMR titrations (Supplemental Fig. 8). However, the amount of $\mathrm{H}_{2} \mathrm{O}_{2}$ measured for reactions with either substrate is very similar (Fig. 8B), thus pointing toward a general disruption in electron delivery and consistent with a disruption in the specificity of the CYP24A1-Adx complex.

\section{Discussion}

Electron transfer by means of a protein interaction with Adx is essential for all reactions catalyzed by mitochondrial $\mathrm{P} 450 \mathrm{~s}$. We previously reported the investigation of clotrimazole-bound CYP24A1-Adx complexes using $\mathrm{P} 450$ isoforms from human, rat, and opossum, which show different regioselectivity for the hydroxylation of the vitamin-D side chain. The different complexes were also shown to rely on distinct secondary binding sites on Adx. Although the previous study presented a correlation between regioselectivity and redox partner recognition, the use of inhibitor in lieu of vitamin-D ligand meant that subtle vitamin-Ddriven modulations of the redox complex were not accessible. In this study, we combine two-dimensional protein NMR, EDC crosslinking, mutagenesis, and functional assays to compare the CYP24A1-Adx complex in the absence of ligand and in the presence of biologic substrate $1,25(\mathrm{OH})_{2} \mathrm{D} 3$, as well as the supplement $1 \alpha(\mathrm{OH}) \mathrm{D} 3$. To remove potential ambiguities from nonconserved species variation of the protein surfaces, we focused entirely on CYP24A1 and Adx from rat, a carbon-24 hydroxylase system.

In the ligand-free state, rat CYP24A1 was found to induce modest differential peak broadening on the HSQC spectra of ${ }^{15} \mathrm{~N}$-Adx (Fig. 2), 
A<smiles>C=C1C(=CC=C2CCCC3(C)C2CCC3[C@H](C)CCCC(C)(C)O)C[C@@H](O)CC1O</smiles>

B
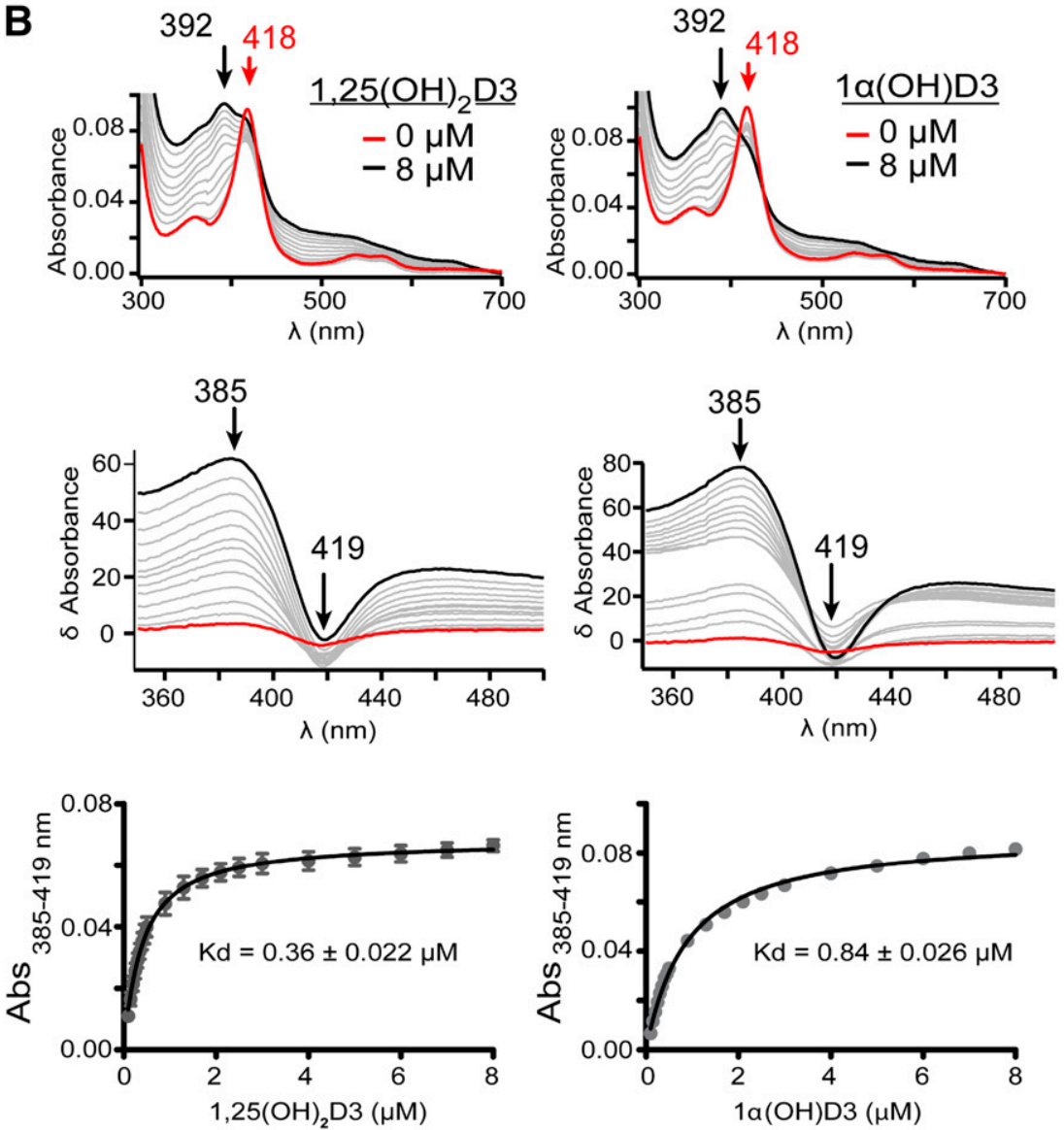

Fig. 6. Spectral binding assays for CYP24A1 vitamin-D ligands. Titrations of $1,25(\mathrm{OH})_{2} \mathrm{D} 3$ and $1 \alpha(\mathrm{OH}) \mathrm{D} 3(\mathrm{~A})$ induce similar high-spin shifts of the Soret band of rat CYP24A1 (B) and bind with similar sub-micromolar affinity. Error bars reflect binding data acquired in triplicate and the Kd calculated error reflects the quality of fit to a hyperbolic binding curve. Error bars for the titration of $1 \alpha(\mathrm{OH}) \mathrm{D} 3$ are smaller than the data icons. with a pattern that points toward the $\alpha$-helix 3 as the principle recognition site. This finding is consistent with prior identification of $\alpha$-helix 3 as a key P450 binding site (Bureik et al., 2005; Heinz et al., 2005; Strushkevich et al., 2011; Peng et al., 2016). Furthermore, as we reported for the mixed-species complexes, disruption of the interaction by point mutations outside of $\alpha$-helix 3 suggests a role for auxiliary contributions from secondary sites, potentially via modulation of the Adx dimer interface. The oxidized form of Adx is known to self-associate into dimers (Pikuleva et al., 2000; Behlke et al., 2007). Among the mutants we generated in rat $\mathrm{Adx}, \mathrm{D} 31 \mathrm{~N}$ is a candidate for involvement in the dimer interface owing to the intermediate and long-range chemical shift perturbations of the mutant ${ }^{15} \mathrm{~N}-\mathrm{HSQC}$ spectrum (Supplemental Figs. 4 and 5).

Substrate-driven modulations of the protein interactions between $\mathrm{P} 450$ s and accessory proteins have previously been reported as a form of regulatory control in the nonmitochondrial P450 enzymes (Estrada et al., 2013, 2014; Bart and Scott, 2017). A salient example is the steroid-metabolizing and multifunctional enzyme CYP17A1, which receives electrons from either a $\mathrm{P} 450$ oxidoreductase (CPR) or the modulator protein cytochrome $b_{5}\left(b_{5}\right)$. Since CPR and $b_{5}$ bind on overlapping surfaces on the proximal side of CYP17A1, the interaction is competitive, with particular CYP17A1 substrates modulating the respective affinities between the redox complexes in a way that correlates with modulation of each reaction (Peng et al., 2016; Duggal et al., 2018). Another example with a more closely related electron delivery scheme to that of mitochondrial P450s is bacterial P450cam (CYP101A1), which binds its cognate redox partner, putidaredoxin, with a higher affinity when bound to substrate (Hollingsworth et al., 2016).

To determine the relevance of similar substrate- or ligand-driven effects as a component of modulating CYP24A1 function, we incorporated both $1,25(\mathrm{OH})_{2} \mathrm{D} 3$ as well as the supplement $1 \alpha(\mathrm{OH}) \mathrm{D} 3$ into the NMR protein-binding assays. $1 \alpha(\mathrm{OH}) \mathrm{D} 3$ bypasses metabolism by the $1 \alpha$-hydroxylase CYP27B1 and is instead rapidly converted into 
A

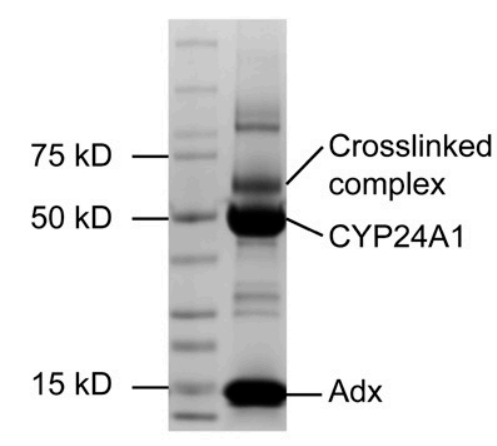

B Adx:CYP24A1 (1:0.2)

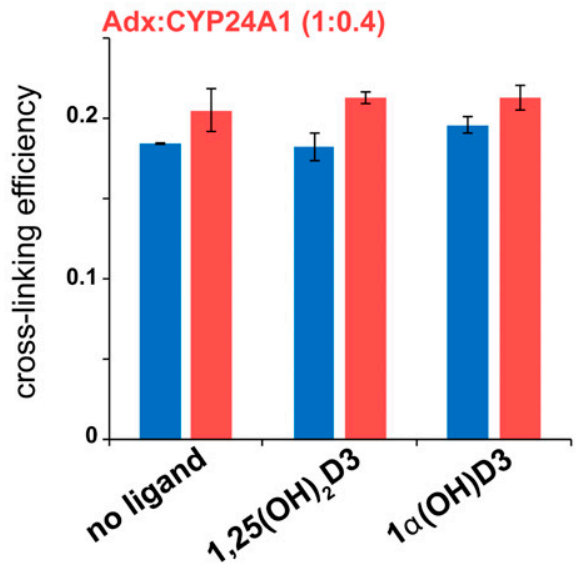

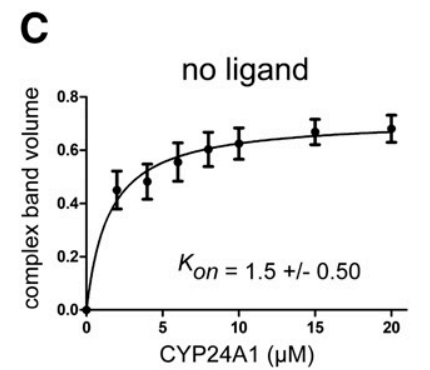

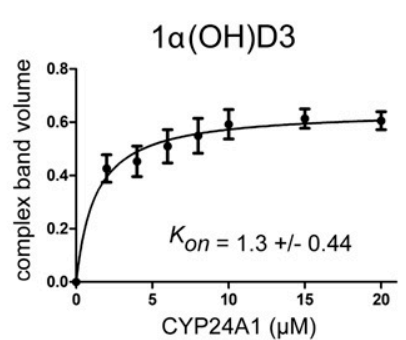

Fig. 7. EDC chemical crosslinking of the CYP24A1-Adx complex. (A) Incubation with EDC results in a CYP24A1-Adx crosslinked product of approximately $65 \mathrm{kDa}$, consistent with a 1:1 complex. To examine crosslinking of the complex with ligand-induced disruption of the complex as observed by NMR, crosslinking was carried out using identical protein ratios as the NMR samples (B), in which the presence of ligand does not appear to disrupt the efficiency of crosslinking. Last, CYP24A1 titrations of the complex as quantified by gel electrophoresis histograms are shown in (C), in which the apparent association constant of the complex is not affected by the presence of vitamin-D ligand.
$1,25(\mathrm{OH})_{2} \mathrm{D} 3$ in liver (Gallacher et al., 1994). Despite having a high affinity for CYP24A1, reconstituted CYP24A1 assays show $1 \alpha(\mathrm{OH}) \mathrm{D} 3$ to be a poor substrate, with turnover rates of less than $5 \%$ of those observed with $1,25(\mathrm{OH})_{2} \mathrm{D} 3$ (Kaufmann et al., 2011). More broadly, modification of the aliphatic side chain in vitamin-D analogs is generally known to reduce catabolism by CYP24A1. For instance, the analogs EB 1089 and 20 -epi-1,25(OH) 2 D3 behave as superagonists in part owing to their sustained metabolic stability (Kissmeyer et al., 1997; Zella et al., 2009; Leyssens et al., 2014).

Here we found that addition of $1,25(\mathrm{OH})_{2} \mathrm{D} 3$ promoted a more focused pattern of peak broadening on $\alpha$-helix 3 of Adx (Fig. 5), consistent with an increase in the specificity of the protein complex greater than was observed without ligand. In contrast, addition of $1 \alpha(\mathrm{OH}) \mathrm{D} 3$ results in disruption of peak broadening, with differential effects distributed on noncontiguous surfaces. We interpret this effect to represent a loss in the specificity of the interaction, with the redox partners probably sampling multiple orientations when bound to the
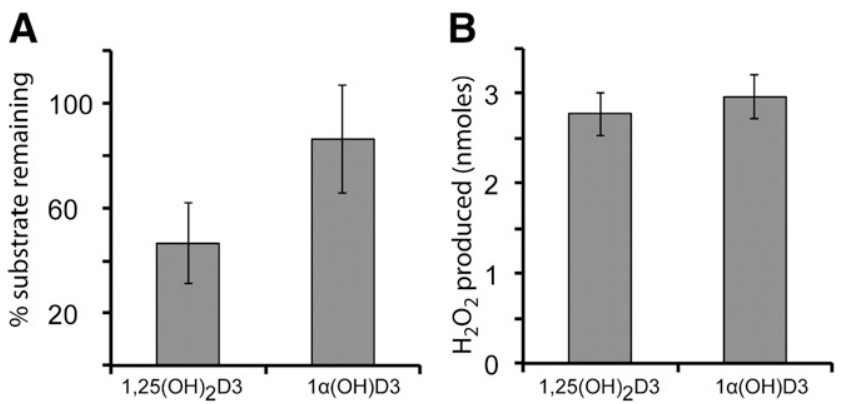

Fig. 8. CYP24A 1 depletion of $1,25(\mathrm{OH})_{2} \mathrm{D} 3$ and $1 \alpha(\mathrm{OH}) \mathrm{D} 3$. Vitamin-D compound depletion was carried out by reconstituted CYP24A1 assays. As expected, $1 \alpha(\mathrm{OH})$ D3 is minimally depleted in comparison with $1,25(\mathrm{OH})_{2} \mathrm{D} 3(\mathrm{~A})$ for a corresponding 5-minute reaction time. However, the amount of $\mathrm{H}_{2} \mathrm{O}_{2}$ produced over the same time period (B) is similar to that measured with the biologic substrate. analog. Loss of specificity is also supported by the observation that the addition of increasing concentrations of CYP24A1 bound to $1 \alpha(\mathrm{OH}) \mathrm{D} 3$ does not appear to induce further complex formation (Fig. 4B). These findings, along with a probable disruption of the functional redox complex (Fig. 8), suggest that proper substrate binding and orientation are necessary for optimal recognition of the electron donor. Furthermore, it underscores the importance of the carbon-25 hydroxyl in substrate orientation in the CYP24A1 active site.

An unexpected finding in this study was the apparent decrease in the relative amount of complex formed between Adx and CYP24A1 bound to $1,25(\mathrm{OH})_{2} \mathrm{D} 3$. This finding is reflected in a one-dimensional spectrum of residue Glu-73 (located on the interaction site of Adx) in Fig. 4A and in the net summary of line broadening shown in Fig. 4B. The implication is that substrate binding appears to reduce the affinity for the electron donor. This outcome stands in contrast to similar NMR studies involving other (nonmitochondrial) P450-redox partner interactions, in which substrate leads to enhanced overall peak broadening (Estrada et al., 2013; Zhang et al., 2015; Bart and Scott, 2017). To verify these effects using a complementary technique, we also carried out a series of EDC chemical crosslinking analyses in which we found no significant difference in crosslinking efficiency between the vitamin-D-bound and ligand-free CYP24A1-Adx complexes. This was in clear contrast to the NMR data, which indicated a ligand-induced loss in the complex. It should be noted, however, that covalent crosslinking and accumulation of the complex may impact binding equilibria in a way that masks small differences in affinity. It should also be noted that a theoretical change in P450-Adx affinity, in particular where multiple recognition sites are involved, might occur independently of changes in functional specificity of the complex. Put another way, changes in ligand-induced specificity, as suggested by the mapping of NMR effects in this study, may or may not be accompanied by changes in protein-protein affinity. Nevertheless, with recognition that substrate-induced changes in $\mathrm{P} 450$ protein-protein interactions are known to occur in microsomal P450s, further investigation of substrate-induced changes in mitochondrial P450s is warranted. 
In summary, these findings provide structural evidence of substrateinduced modulation of the CYP24A1 redox-binding surface, as represented by differential NMR peak broadening of the Adx surface. This feature of $\mathrm{P} 450$ function has not previously been demonstrated for a mitochondrial member of the P450 family. With respect to vitamin-D metabolism, these findings also provide evidence that the specificity of vitamin-D recognition, particularly as determined by the carbon- 25 hydroxyl of $1,25(\mathrm{OH})_{2} \mathrm{D} 3$, also confers specificity on the CYP24A1Adx interaction. More broadly, this work also provides a structural basis for low substrate turnover of side-chain-modified vitamin-D analogs.

\section{Acknowledgments}

The authors thank Dr. Richard Browne and Sonia Bhattacharya from the Department of Biotechnical and Clinical Laboratory Sciences, University at Buffalo, for their assistance in optimizing the preparation of vitamin-D liquid chromatography samples used to resolve vitamin-D substrates.

\section{Authorship Contributions}

Participated in research design: Kumar, Estrada.

Conducted experiments: Kumar.

Performed data analysis: Kumar.

Wrote or contributed to the writing of the manuscript: Kumar, Estrada.

\section{References}

Bart AG and Scott EE (2017) Structural and functional effects of cytochrome $b_{5}$ interactions with human cytochrome P450 enzymes. J Biol Chem 292:20818-20833.

Bax A and Ikura M (1991) An efficient 3D NMR technique for correlating the proton and 15N backbone amide resonances with the alpha-carbon of the preceding residue in uniformly $15 \mathrm{~N} /$ 13C enriched proteins. J Biomol NMR 1:99-104.

Beckman MJ, Tadikonda P, Werner E, Prahl J, Yamada S, and DeLuca HF (1996) Human 25hydroxyvitamin D3-24-hydroxylase, a multicatalytic enzyme. Biochemistry 35:8465-8472.

Behlke J, Ristau O, Müller EC, Hannemann F, and Bernhardt R (2007) Self-association of adrenodoxin studied by using analytical ultracentrifugation. Biophys Chem 125:159-165.

Bureik M, Zöllner A, Schuster N, Montenarh M, and Bernhardt R (2005) Phosphorylation of bovine adrenodoxin by protein kinase CK2 affects the interaction with its redox partner cytochrome P450scc (CYP11A1). Biochemistry 44:3821-3830.

Constantine KL, Goldfarb V, Wittekind M, Friedrichs MS, Anthony J, Ng SC, and Mueller L (1993) Aliphatic $1 \mathrm{H}$ and $13 \mathrm{C}$ resonance assignments for the 26-10 antibody VL domain derived from heteronuclear multidimensional NMR spectroscopy. J Biomol NMR 3:41-54.

Delaglio F, Grzesiek S, Vuister GW, Zhu G, Pfeifer J, and Bax A (1995) NMRPipe: a multidimensional spectral processing system based on UNIX pipes. J Biomol NMR 6:277-293.

Duggal R, Denisov IG, and Sligar SG (2018) Cytochrome $b_{5}$ enhances androgen synthesis by rapidly reducing the CYP17A1 oxy-complex in the lyase step. FEBS Lett 592:2282-2288.

Estrada DF (2018) The cytochrome P450 24A1 interaction with adrenodoxin relies on multiple recognition sites that vary among species. $J$ Biol Chem 293:4167-4179.

Estrada DF, Laurence JS, and Scott EE (2013) Substrate-modulated cytochrome P450 17A1 and cytochrome b5 interactions revealed by NMR. J Biol Chem 288:17008-17018.

Estrada DF, Skinner AL, Laurence JS, and Scott EE (2014) Human cytochrome P450 17A1 conformational selection: modulation by ligand and cytochrome b5. J Biol Chem 289: $14310-14320$.

Ewen KM, Kleser M, and Bernhardt R (2011) Adrenodoxin: the archetype of vertebrate-type [2Fe2S] cluster ferredoxins. Biochim Biophys Acta 1814:111-125.

Gallacher SJ, Cowan RA, Fraser WD, Logue FC, Jenkins A, and Boyle IT (1994) Acute effects of intravenous 1 alpha-hydroxycholecalciferol on parathyroid hormone, osteocalcin and calcitriol in man. Eur J Endocrinol 130:141-145.

Grzesiek S, Döbeli H, Gentz R, Garotta G, Labhardt AM, and Bax A (1992) 1H, 13C, and 15N NMR backbone assignments and secondary structure of human interferon-gamma. Biochemistry 31:8180-8190

Grzesiek S, Vuister GW, and Bax A (1993) A simple and sensitive experiment for measurement of JCC couplings between backbone carbonyl and methyl carbons in isotopically enriched proteins. J Biomol NMR 3:487-493.
Hamamoto H, Kusudo T, Urushino N, Masuno H, Yamamoto K, Yamada S, Kamakura M, Ohta M, Inouye K, and Sakaki T (2006) Structure-function analysis of vitamin D 24-hydroxylase (CYP24A1) by site-directed mutagenesis: amino acid residues responsible for species-based difference of CYP24A1 between humans and rats. Mol Pharmacol 70:120-128.

Heinz A, Hannemann F, Müller JJ, Heinemann U, and Bernhardt R (2005) The interaction domain of the redox protein adrenodoxin is mandatory for binding of the electron acceptor CYP11A1, but is not required for binding of the electron donor adrenodoxin reductase. Biochem Biophys Res Commun 338:491-498.

Holick MF (2007) Vitamin D deficiency. N Engl J Med 357:266-281.

Hollingsworth SA, Batabyal D, Nguyen BD, and Poulos TL (2016) Conformational selectivity in cytochrome P450 redox partner interactions. Proc Natl Acad Sci USA 113:8723-8728.

Johnson BA (2004) Using NMRView to visualize and analyze the NMR spectra of macromolecules. Methods Mol Biol 278:313-352.

Kaufmann M, Prosser DE, and Jones G (2011) Bioengineering anabolic vitamin D-25-hydroxylase activity into the human vitamin D catabolic enzyme, cytochrome P450 CYP24A1, by a V391L mutation. J Biol Chem 286:28729-28737.

Kissmeyer AM, Binderup E, Binderup L, Mørk Hansen C, Andersen NR, Makin HL, Schroeder NJ, Shankar VN, and Jones G (1997) Metabolism of the vitamin D analog EB 1089: identification of in vivo and in vitro liver metabolites and their biological activities. Biochem Pharmaco 53:1087-1097.

Kostic M, Pochapsky SS, Obenauer J, Mo H, Pagani GM, Pejchal R, and Pochapsky TC (2002) Comparison of functional domains in vertebrate-type ferredoxins. Biochemistry 41: 5978-5989.

Laskowski RA, Rullmannn JA, MacArthur MW, Kaptein R, and Thornton JM (1996) AQUA and PROCHECK-NMR: programs for checking the quality of protein structures solved by NMR. J Biomol NMR 8:477-486.

Leyssens C, Verlinden L, and Verstuyf A (2014) The future of vitamin D analogs. Front Physiol 5: 122.

Peng HM and Auchus RJ (2017) Molecular recognition in mitochondrial cytochromes P450 that catalyze the terminal steps of corticosteroid biosynthesis. Biochemistry 56:2282-2293.

Peng HM, Im SC, Pearl NM, Turcu AF, Rege J, Waskell L, and Auchus RJ (2016) Cytochrome b5 activates the 17,20-lyase activity of human cytochrome P450 17A1 by increasing the coupling of NADPH consumption to androgen production. Biochemistry 55:4356-4365.

Pikuleva IA, Tesh K, Waterman MR, and Kim Y (2000) The tertiary structure of full-length bovine adrenodoxin suggests functional dimers. Arch Biochem Biophys 373:44-55.

Prosser DE, Kaufmann M, O'Leary B, Byford V, and Jones G (2007) Single A326G mutation converts human CYP24A1 from 25-OH-D3-24-hydroxylase into -23-hydroxylase, generating 1alpha,25-(OH)2D3-26,23-lactone. Proc Natl Acad Sci USA 104:12673-12678.

Ryan JW, Anderson PH, Turner AG, and Morris HA (2013) Vitamin D activities and metabolic bone disease. Clin Chim Acta 425:148-152.

Siu-Caldera ML, Zou L, Ehrlich MG, Schwartz ER, Ishizuka S, and Reddy GS (1995) Human osteoblasts in culture metabolize both 1 alpha, 25-dihydroxyvitamin D3 and its precursor 25-hydroxyvitamin D3 into their respective lactones. Endocrinology 136:4195-4203.

Strushkevich N, MacKenzie F, Cherkesova T, Grabovec I, Usanov S, and Park HW (2011) Structural basis for pregnenolone biosynthesis by the mitochondrial monooxygenase system. Proc Natl Acad Sci USA 108:10139-10143.

Waterhouse A, Bertoni M, Bienert S, Studer G, Tauriello G, Gumienny R, Heer FT, de Beer TAP, Rempfer C, Bordoli L, et al. (2018) SWISS-MODEL: homology modelling of protein structures and complexes. Nucleic Acids Res 46 (W1):W296-W303.

Weiss R, Brachais L, Löhr F, Hartleib J, Bernhardt R, and Rüterjans H (2000) Assignment of $1 \mathrm{H}$ $13 \mathrm{C}$ and $15 \mathrm{~N}$ signals of bovine adrenodoxin. J Biomol NMR 17:355-356.

Weissgerber TL, Savic M, Winham SJ, Stanisavljevic D, Garovic VD, and Milic NM (2017) Data visualization, bar naked: a free tool for creating interactive graphics. J Biol Chem 292: 20592-20598.

Wishart DS and Sykes BD (1994) The 13C chemical-shift index: a simple method for the identification of protein secondary structure using $13 \mathrm{C}$ chemical-shift data. J Biomol NMR 4: 171-180.

Yang J, Yan R, Roy A, Xu D, Poisson J, and Zhang Y (2015) The I-TASSER Suite: protein structure and function prediction. Nat Methods 12:7-8.

Zella LA, Meyer MB, Nerenz RD, and Pike JW (2009) The enhanced hypercalcemic response to 20-epi-1,25-dihydroxyvitamin D3 results from a selective and prolonged induction of intestinal calcium-regulating genes. Endocrinology 150:3448-3456.

Zhang M, Le Clair SV, Huang R, Ahuja S, Im SC, Waskell L, and Ramamoorthy A (2015) Insights into the role of substrates on the interaction between cytochrome b5 and cytochrome P450 2B4 by NMR. Sci Rep 5:8392.

Address correspondence to: Dr. Fernando Estrada, Department of Biochemistry, Jacobs School of Medicine and Biomedical Science, University at Buffalo, Buffalo, NY 14203. E-mail: dfestrad@buffalo.edu 